\title{
Activity and health monitoring systems
}

\author{
Béla-Csaba Simon ${ }^{(1)}$, Stefan Oniga ${ }^{(1),(2)}$, Iuliu Alexandru Pap ${ }^{(2)}$ \\ (1) Intelligent Embedded Systems Research Laboratory, Department of IT Systems and Networks, Faculty of Informatics, \\ University of Debrecen, Hungary \\ (2) Electric, Electronic and Computer Engineering Department, \\ Technical University of Cluj-Napoca, North University Center of Baia Mare, Romania \\ iuliu.alexandru@gmail.com
}

\begin{abstract}
This paper presents an Open Platform Activity and health monitoring systems which are also called e-Health systems. These systems measure and store parameters that reflect changes in the human body. Due to continuous monitoring (e.g. in rest state and in physical effort state), a specialist can learn about the individual's physiological parameters. Because the human body is a complex system, the examiner can notice some changes within the body by looking at the physiological parameters. Six different sensors ensure us that the patient's individual parameters are monitored. The main components of the device are: A Raspberry Pi 3 small single-board computer, an e-Health Sensor Platform by Cooking-Hacks, a Raspberry Pi to Arduino Shields Connection Bridge and a 7-inch Raspberry $P i 3$ touch screen. The processing unit is the Raspberry Pi 3 board. The Raspbian operating system runs on the Raspberry $\mathrm{Pi} 3$, which provides a solid base for the software. Every examination can be controlled by the touch screen. The measurements can be started with the graphical interface by pressing a button and every measured result can be represented on the GUI's label or on the graph. The results of every examination can be stored in a database. From that database the specialist can retrieve every personalized data.
\end{abstract}

Keywords-e-Health, activity monitoring, health monitoring, human activity recognition, patient monitoring, Internet of Things.

\section{INTRODUCTION}

A lot of electronic products contain embedded systems. They surround us, entertain and even improve our quality of life. For example, they are present in cars, various alarm systems, mobile devices, some household electronics and medical devices. The foundation of an e-Health system is an embedded system. The e-Health is nothing more than the use of information and communication technologies for prevention, supervision, self-care, research and development. There are many benefits in applying the e-Health concept: the paper-based healthcare card will be eliminated, the health service will be much more dynamic and swift. There are several types of activities and health monitoring systems, as interest in e-Health is growing [1]. At every constantly working system we have to pay attention to the Big Data phenomenon. This large amount of data should be processed and classified. Various learning algorithms can provide predictions based on patient data.

From a significant number of studies [2-8] we can observe the importance of using assistive devices or robots to improve our lives. Health monitoring systems are one of the best candidates for testing human-machine interaction.
The aim of our research is to provide a functional software platform capable of collecting data from sensors with minimal pre-processing.

A similar system is described in [9], where the software components follow a modular approach, being written in multiple languages. In comparison, our software implementation uses a single programming language and the user interface is compatible with all Linux distributions.

By studying some other approaches [9-13] to health and physical activity monitoring, we were able to obtain a better understanding of how to measure and use the sensor data. Major universities and research centers have conducted research related to e-Health systems. The Dutch University of Twente put e-Health in a slightly different perspective. They developed a platform which combines psychology, healthcare and technology. The study presented different kinds of physiological changes that the psychological states can evoke. We can mention another project which is called MySignals. This project includes multiple physiological examinations.

The project started with integrating microelectronics and sensors into a device. We used the following sensors: Pulse Oximeter, ECG, Airflow, Thermometer, Accelerometer, Galvanic Skin Response.

This system can be used by the elderly people, the sick people or people with disabilities. The kind of lifestyle a person is experiencing and the kind of diseases he or she is susceptible to can be inferred from the measured data. For easy handling we developed a graphical user interface. Through a 7-inch touch screen we can control (start or stop) and we can see (on a GUI's label or on a graph) the measurements by pressing some buttons on the graphical user interface. The examiner can use the sensors one by one or all six sensors at the same time. Due to the aforementioned structure, it performs several tens of measurements per second. These datasets describe the changes related to the physiological state of the patient.

There are two modes: Offline and Online. In offline mode we can measure the body parameters without storing the measured data in the database. In online mode we need to complete a surface with our personal data. From that moment every measured data will be personalized. So we can identify the person to whom the data belongs to. The measured data will be saved in a relational database. At this point we have a stored historical data set from which we can determine the state of our patient's health. If we connect a webpage to this database, we can view our personal analysis. 
The device can be portable by using a battery, but it can also work with an adapter. This project consists of two parts. In the first part we assembled the hardware and in the second part we developed the software. During the development we focused on functions like measuring physiological parameters, plotting the data and storing historical data in a database.

\section{HARDWARE CONFIGURATION}

We used an e-Health Sensor Platform V2.0 which was compatible with Arduino and Raspberry Pi. This platform allowed us to do biometric and medical examinations with each sensor. The processing unit was a Raspberry Pi 3. We connected the e-Health Sensor Platform directly to Arduino Uno, but our goal was to use the Raspberry Pi's hardware resources. For this reason we used a Connection Bridge which connected the Raspberry Pi to the e-Health Sensor Platform.

We plugged a $5 \mathrm{~V}$ DC $2.5 \mathrm{~A}$ adapter into the micro USB input of the touch screen controller. From that moment, with two jumper wires the controller could share the VCC and the GND. The two wires were connected to the e-Health platform VCC pin-in and GND pin-in. Raspberry Pi 3 Board was then powered through the Connection Bridge. So at this stage I had a complete hardware system that I could attach different sensors to. Thereafter we connected the screen controller's DSI ribbon cable to Raspberry $\mathrm{Pi} 3$. The communication between Raspberry $\mathrm{Pi} 3$ and e-Health is realized by GPIO.

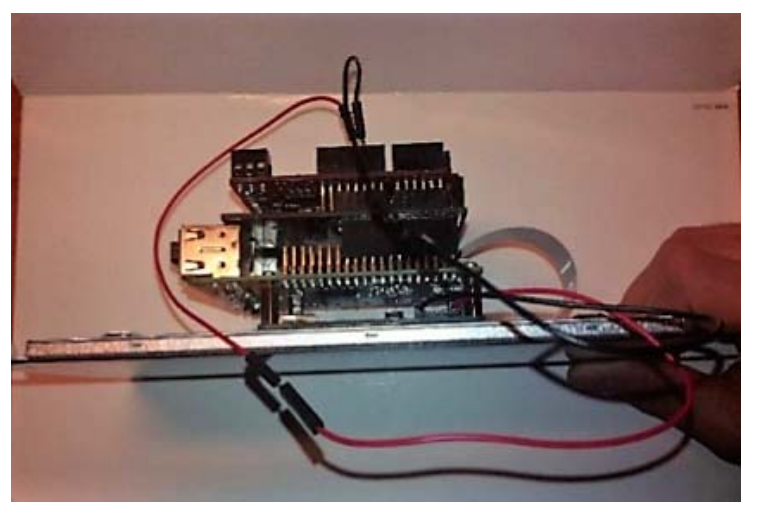

Fig. 1. The assembled Raspberry Pi3, Connection Bridge and e-Health Sensor Plaform

\section{A. Operating System}

In order to write a code in high level programming language we installed a Raspbian operating system on our SD card. We realized this on a PC with a Raspbian image file and the Win32 Disk Imager program. The installation consisted in writing the Raspbian raw disk image to the MicroSD card by using the Win32 Disk Imager program. The result was a bootable memory card.

\section{B. The Pulse Oximeter}

The pulse oximeter is a non-invasive procedure for signaling the saturation of functional hemoglobin of arterial oxygen. Oxygen saturation (saturation) is the amount of oxygen dissolved in the blood based on the detection of deoxyhemoglobin and hemoglobin. We connected the
Quirumed Contec CMS50D+ pulse oximeter to the Raspberry Pi 3 USB-port.

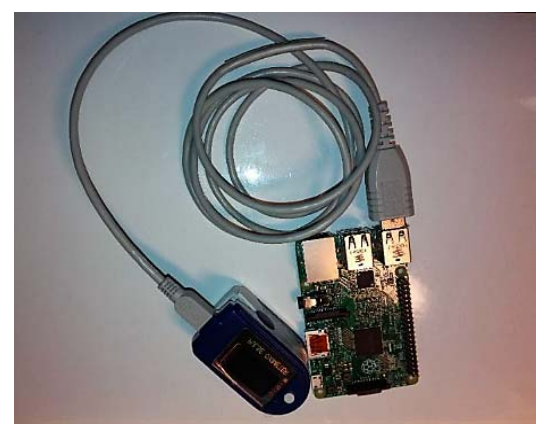

Fig. 2. The Raspberry Pi3 and Pulse Oximeter

Pulse oximeter measuring range and accuracy:

- $\quad$ SPO2: $35 \%-99 \%, \pm 2 \%$,

- $\quad$ Pulse: 30 - 250 BPM, \pm 2 BPM.

The pulse oximeter provides $60 \mathrm{~Hz}$ data. In terms of coding, real-time communication is straightforward, because the communication protocol is one-way only. We attached the device to our finger and after pressing a button, it started to measure our pulse.

\section{Electrocardiogram (ECG)}

Three electrodes are required to be connected to the patient. One electrode is positive, the second is negative, and the third is neutral:
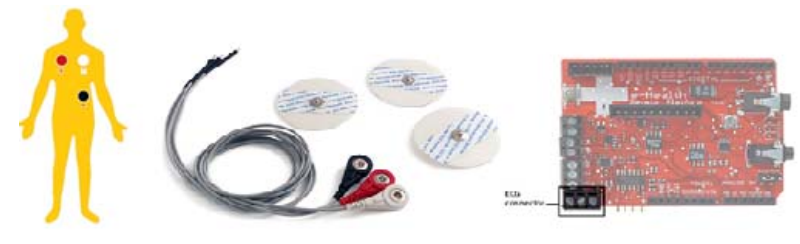

Fig. 3. ECG connection

These three measuring points form an equilateral triangle around the heart. This is called the Einthoven triangle. The electrodes are color-coded because their positions cannot be interchanged.

\section{Airflow Sensor}

The airflow sensor could detect abnormal respiration rate and a wide range of physiological instability. The Airflow sensor provides information about our patient whether our patient is suffering from hypoxia or apnea state. A healthy adult breath rate is $15-30$ breaths per minute. The airflow sensor has two pins. One is positive, another one is negative. These pins were connected directly to the e-Health platform.

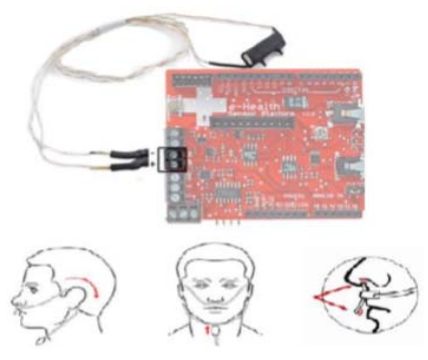

Fig. 4. The Airflow connection 


\section{E. Body Temperature Measurement}

Our measurement depends on which part of the body we perform the measurement, what physical activity is performed during the measurement and at what stage of the day we perform the measurement. The body temperature is $37^{\circ} \mathrm{C}$. It may fluctuate $0.5^{\circ} \mathrm{C}$ for a healthy adult. The body temperature is lower in the morning and in the afternoon, but it is higher in the evening because our activity changes. The temperature sensor can be connected to e-health sensor with a simple jack connector.

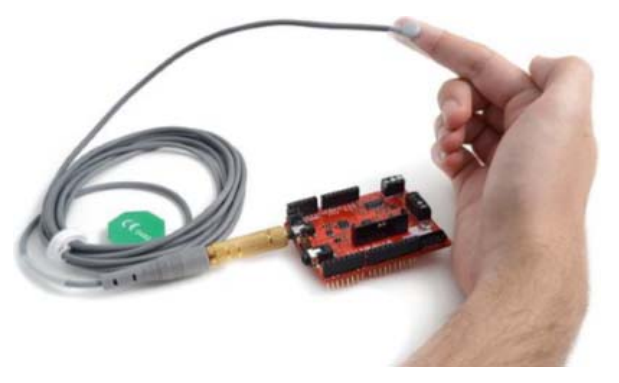

Fig. 5. Temperature sensor connection

\section{F. Accelerometer}

In many cases, it is important to monitor the patient's body position and movement. For example, analysis of sleep patterns will help determine sleep quality and sleep habits.

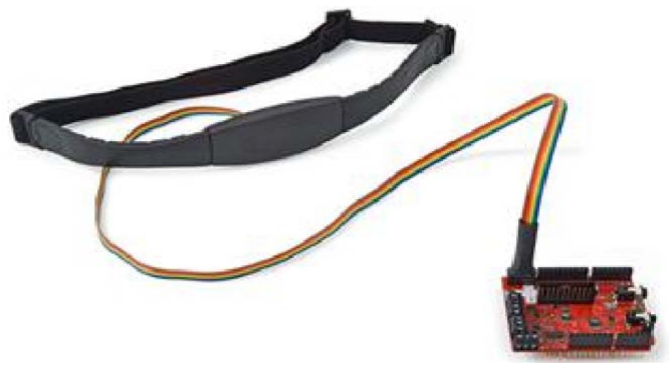

Fig. 6. The accelerometer connection

The accelerometer detects the patient in the following positions:

1. Sitting / standing,

2. Face upward,

3. Face down,

4. Lying on the right side,

5. Lying on the left side.

\section{G. Galvanic Skin Response}

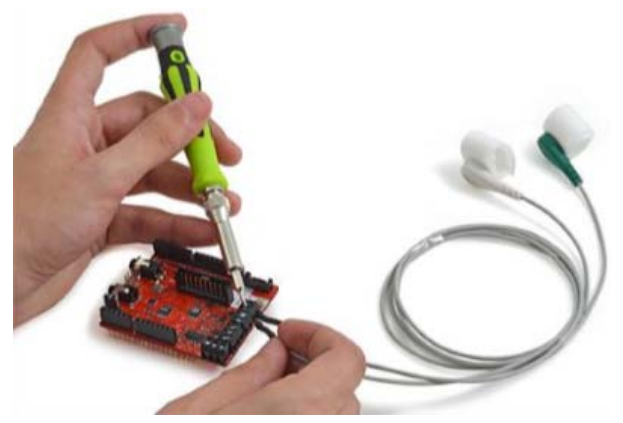

Fig. 7. The GSR connection
The electrical resistance of the skin is also called the Galvanic Skin Resistance. Essentially, this is the measurement of skin conductivity, which is directly proportional to the moisture of the skin. This is interesting because the sweat glands are controlled by the sympathetic nervous system, so that in the case of stronger emotional moments the electrical resistance of the skin may change. We can interpret the conductivity of the skin as a psychic or physiological excitement.

\section{The Device Software}

\section{A. Communication between our health device and remote user}

We changed the data via a database. When we insert a row into the database a remote user can retrieve the examination result in real time [14].

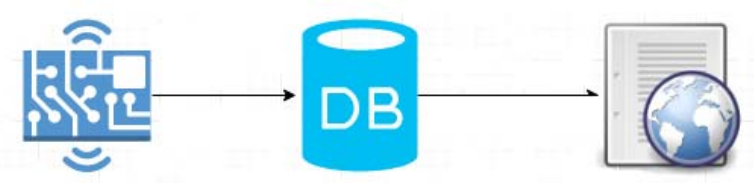

Fig. 8. Remote Connect

\section{B. Graphical User Interface}

The graphical interface facilitates usability. The software has features that make interacting with the device's user interface very trivial. The user feels that the device is a simple tablet.

There is a menu where the available sensors are presented. We only need to touch these, and the examination will start. After selecting the preferred examination the measurement begins. The measured values will be displayed in real time. By touching the plotting button a window will pop up that represents a real time plotter. If we want to store historical data, we only need to touch the record button. Our data will be stored in a database and a specialist can make a diagnosis.

Another important function is the Summary function. With this function the device is able to take multiple examinations at the same time.

\section{Personalized data}

The program starts with a personalized interface. Here the interface is looking for the patient's personal data: name, birth date, height, weight. After touching the OK button the data will be inserted in our database. Thereafter a list of available sensors will pop up.

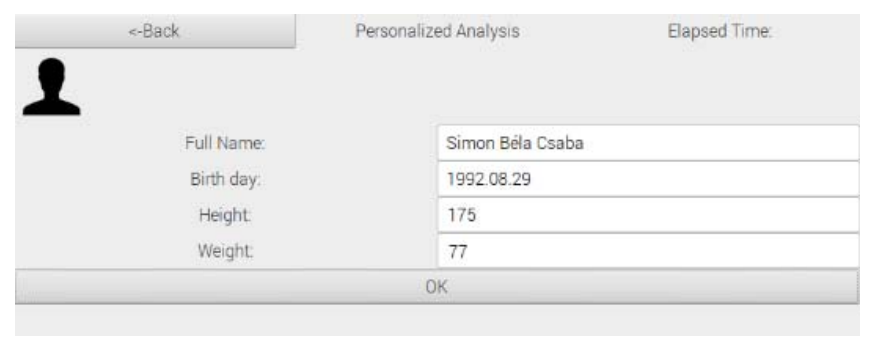

Fig. 9. Personalized GUI 


\section{Select Sensors Interface}

By touching the sensor buttons the user can start an examination and by touching the Back button the user can also stop the examination.

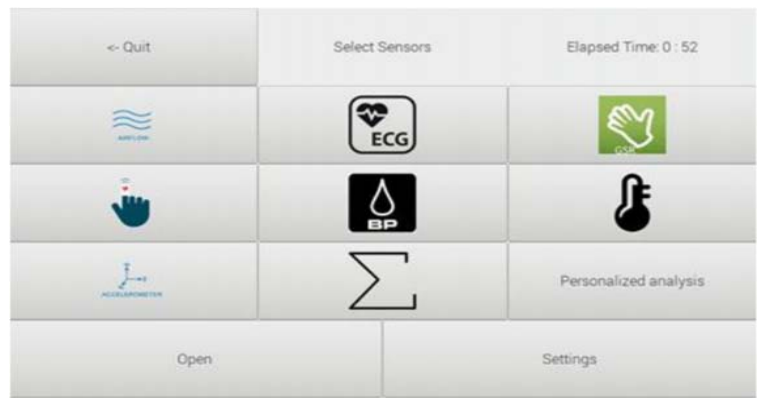

Fig. 10. Select Sensors GUI

\section{E. Sensor's Window}

Every sensor has a specific window. The plotting function and the record function belong to these windows. So the user can choose the visualization mode. The plotting will happen in real time so the state of graph is constantly changing.
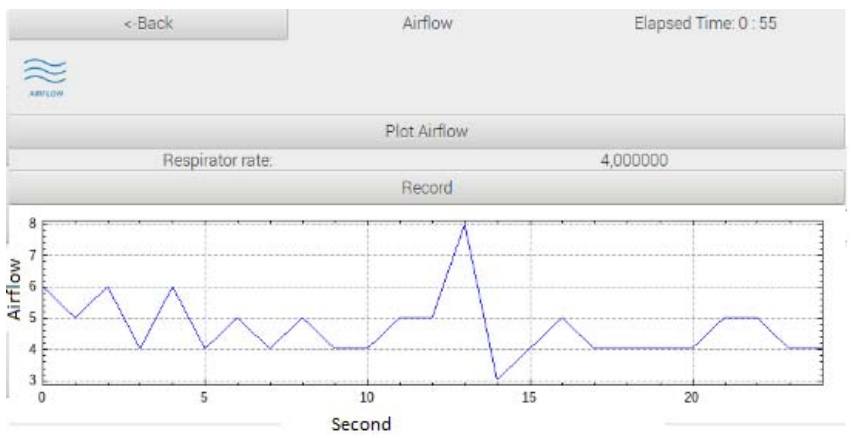

Fig. 11. Sensor's Window

The $\mathrm{X}$ axis represents the time in seconds. The $\mathrm{Y}$ axis represents the measured values. When the user presses the Record button the actual date will be stored in the database. Every patient will get a new row in our Person table and every sensor is represented with a particular table.

\section{F. Summarize Interface}

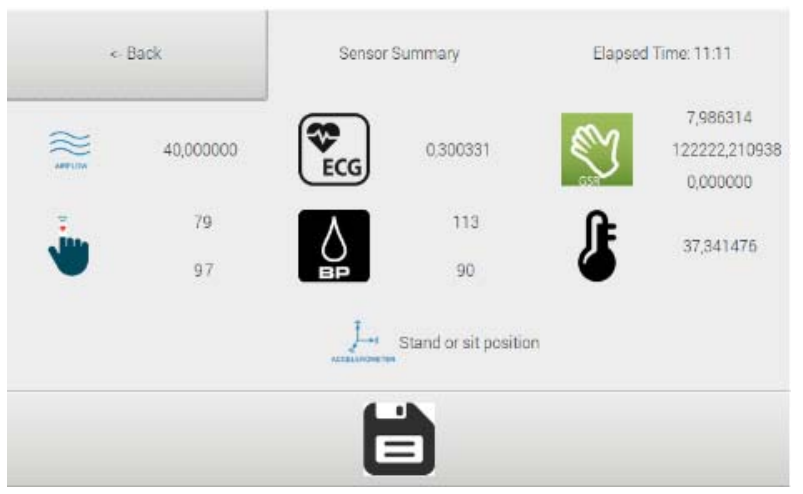

Fig. 12. Summary Window
With this function the device is able to do simultaneous examinations. Every sensor measurement is displayed on the interface of these functions.

\section{CONCLUSION}

In the research presented in this paper we succeeded to assemble the hardware modules and develop the software.

The system we built provides eHealth monitoring functions such as measuring, displaying and storing multiple health parameters.

During our research we had to overcome some difficulties like handling large amounts of data in short time frames.

This work was made as a part of the ongoing research that takes place in the Intelligent Embedded Systems Research Laboratory at the University of Debrecen, Hungary, with the aims to assist elderly or sick people in everyday activities using the latest assistive technologies.

\section{REFERENCES}

[1] L. Gatzoulis, I. Iakovidis, "Wearable and Portable eHealth Systems", IEEE Engineering in Medicine and Biology Magazine, vol. 26, no. 5, pp. 1-2, 2007.

[2] A. Alexan, A. Osan, S. Oniga, "Personal assistant robot," in Proceedings of 2012 IEEE 18th International Symposium for Design and Technology in Electronic Packaging, Alba Iulia, Romania, 2012.

[3] A. Alexan, A. Osan, S. Oniga, "AssistMe robot, an assistance robotic platform," Carpathian Journal of Electronic and Computer Engineering, vol. 5, no. 1, pp. 1-4, 2012.

[4] I. Orha, S. Oniga, "Assistance and telepresence robots: a solution for elderly people," Carpathian Journal of Electronic and Computer Engineering, vol. 5, no. 1, pp. 87-90, 2012.

[5] J. Sütő, S. Oniga, "Remote controlled data collector robot," Carpathian Journal of Electronic and Computer Engineering, vol. 5, no. 1, pp. 117-120, 2012.

[6] E. Ackerman, "Suitable Technologies Introduces Beam Remote Presence System," IEEE Spectrum Robotics News, 26 September 2012.

[7] Ha M. Do, C. Mouser, W. Sheng, “An Open Platform Telepresence Robot with Natural Human Interface" Proceedings of the 2013 IEEE International Conference on Cyber Technology in Automation, Control and Intelligent Systems May 26-29, 2013, Nanjing, China.

[8] D. Feil-Seifer, M. Mataric, "Human-robot interaction," in Encyclopedia of Complexity and System Science, new York, Springer, 2009, pp. 4643-4659.

[9] I. A. Pap, S. Oniga, I. Orha, A. Alexan, "IoT-Based eHealth Data Acquisition System", International Conference on Automation, Quality and Testing, Robotics, Cluj-Napoca, Romania, 2018.

[10] P. Freedson, H. Bowles, R. Troiano, W. Haskell, "Assessment of Physical Activity Using Wearable Monitors", Medicine \& Science in Sports \& Exercise, vol. 44, pp. S1-S4, 2012.

[11] V. Dupourqué, "DOMEO, an Open Robotic Platform for Cognitive and Physical Personalized Homecare Services," in Workshop PAL, Sophia Antipolis, France, 2011.

[12] A. Mataric, A. Okamura, H. Christensen, "A Research Roadmap for Medical and Healthcare Robotics," Arlington, VA, 2008.

[13] M. Heerink, B. Kröse, V. Evers, B. Wielinga, "Assessing Acceptance of Assistive Social Agent Technology by Older Adults: the Almere Model," International Journal of Social Robotics, vol. 2, no. 4, pp. 361-375, 2010.

[14] J. Lu, S. Zhang, "Master Thesis - E-health Web Application Framework and Platform Based On The Cloud Technology", School of Health and Society Department Design and Computer Science, 2013 . 